\title{
Medicago PHYA promotes flowering, primary stem elongation and expression of flowering time genes in long days
}

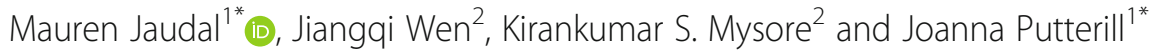

\begin{abstract}
Background: Flowering time is an important trait for productivity in legumes, which include many food and fodder plants. Medicago truncatula (Medicago) is a model temperate legume used to study flowering time pathways. Like Arabidopsis thaliana (Arabidopsis), its flowering is promoted by extended periods of cold (vernalization, V), followed by warm long day (LD) photoperiods. However, Arabidopsis flowering-time genes such as the FLOWERING LOCUS C (FLC)/ MADS AFFECTING FLOWERING (MAF) clade are missing and CONSTANS-LIKE (COLIKE) genes do not appear to have a role in Medicago or Pisum sativum (pea). Another photoperiodic regulator, the red/far red photoreceptor PHYTOCHROME A (PHYA), promotes Arabidopsis flowering by stabilizing the CO protein in LD. Interestingly, despite the absence of CO-LIKE function in pea, PsPHYA plays a key role in promoting LD photoperiodic flowering and plant architecture. Medicago has one homolog of PHYA, MtPHYA, but its function is not known.

Results: Genetic analysis of two MtPHYA Tnt1 insertion mutant alleles indicates that MtPHYA has an important role in promoting Medicago flowering and primary stem elongation in VLD and LD and in perception of far-red wavelengths in seedlings. MTPHYA positively regulates the expression of MtE1-like (MtE1L), a homologue of an important legume-specific flowering time gene, E1 in soybean and other Medicago LD-regulated flowering-time gene homologues, including the three FLOWERING LOCUS T-LIKE (FT-LIKE) genes, MtFTa1, MtFTb1 and MtFTb2 and the two FRUITFULL-LIKE (FUL-LIKE) genes MIFULa and MtFULb. MTPHYA also modulates the expression of the circadian clock genes, GIGANTEA (GI) and TIMING OF CAB EXPRESSION 1a (TOCIa). Genetic analyses indicate that Mtphya-1 Mte1/ double mutants flowered at the same time as the single mutants. However, Mtphya-1 Mtfta1 double mutants had a weak additive effect in delaying flowering and in reduction of primary axis lengths beyond what was conferred by either of the single mutants.

Conclusion: MtPHYA has an important role in LD photoperiodic control of flowering, plant architecture and seedling de-etiolation under far-red wavelengths in Medicago. It promotes the expression of LD-induced flowering time genes and modulates clock-related genes. In addition to MtFTa1, MtPHYA likely regulates other targets during LD floral induction in Medicago.
\end{abstract}

Keywords: PHYA, Photoperiodic flowering time, Medicago, Arabidopsis, Legume, FTa1, E1, FUL, SOC1, Primary stem elongation

\footnotetext{
* Correspondence: m.jaudal@auckland.ac.nz; j.putterill@auckland.ac.nz

${ }^{1}$ The Flowering Lab, School of Biological Sciences, University of Auckland, Auckland, New Zealand

Full list of author information is available at the end of the article
}

\section{$\triangle B M C$}

(c) The Author(s). 2020 Open Access This article is licensed under a Creative Commons Attribution 4.0 International License, which permits use, sharing, adaptation, distribution and reproduction in any medium or format, as long as you give appropriate credit to the original author(s) and the source, provide a link to the Creative Commons licence, and indicate if changes were made. The images or other third party material in this article are included in the article's Creative Commons licence, unless indicated otherwise in a credit line to the material. If material is not included in the article's Creative Commons licence and your intended use is not permitted by statutory regulation or exceeds the permitted use, you will need to obtain permission directly from the copyright holder. To view a copy of this licence, visit http://creativecommons.org/licenses/by/4.0/ The Creative Commons Public Domain Dedication waiver (http://creativecommons.org/publicdomain/zero/1.0/) applies to the data made available in this article, unless otherwise stated in a credit line to the data. 


\section{Background}

Legumes are the third largest group of plants and the second most important crops after the cereals, with flowering time a key factor in determining their productivity and yield [1-4]. Medicago truncatula (Medicago) is a model nitrogen-fixing legume and a diploid, self-fertile annual forage plant [5]. It is related to important temperate legume forages and foods including Medicago sativa (alfalfa), Trifolium species (clovers), Pisum sativum (garden pea), Cicer arietinum (chickpeas) and Lens culinaris (lentils). Major Medicago genomic resources include genome sequences and large collections of mutants including Tnt1 retroelement insertion lines [5-7]. These are accompanied by a searchable flanking sequence tag database facilitating forward and reverse genetics to analyze flowering time control [6-9].

Like the winter annual ecotypes of Arabidopsis thaliana (Arabidopsis), Medicago is a temperate-climate plant whose flowering time is promoted by long day (LD) photoperiods and by vernalization - an extended exposure to winter cold. This ensures that flowering occurs in the lengthening warm days of spring only after winter has passed [10]. However, interestingly, the key regulatory genes known to be targets of the Arabidopsis vernalization pathway - the repressor FLOWERING LOCUS C (FLC) and associated MADS AFFECTING FLOWERING (MAF) clade are missing in Medicago $[4,8]$.

In addition, while CONSTANS (CO) has an important role in photoperiodic control of flowering in Arabidopsis activating the potent stimulator of flowering FLOWERING LOCUS T (FT) in leaves in LD, Medicago CO-like genes do not appear to be involved in flowering time control [11]. Mt co-like Tht1 insertion mutants flower like wild-type [11]. Similarly, the transcript levels of MtCO-like genes are not altered by the overexpression of a Medicago CYCLING DOF FACTOR-like gene, $M t C D F d 1 \_1$ that nevertheless leads to a delay in Medicago flowering in LD [12]. Similar results were obtained in the analysis of pea flowering time mutants that affect photoperiodic flowering and/or circadian clock function such as late2 (Pscdfc1) [13], late1 (Psgigantea (gi)) [14] and dne (die neutralis, Pself4) [4, 15]. These mutations alter pea $F T$-like gene expression, but not the expression of the closest pea relative to CO, PsCOLa.

Other candidate components of Medicago floweringtime pathways including FT-LIKE genes have also been investigated and are implicated in flowering time control. MtFTa1 is a key regulator of flowering in Medicago. It is expressed predominantly in leaves where expression is elevated by exposure to the floral inductive conditions of vernalization followed by LD. In addition, Mtfta 1 Tnt1 insertion mutants were late flowering, while over expression of MtFTa1 accelerated flowering [16]. The expression of MtFTa1 and the two other LD-induced
Medicago FT-like genes, MtFTb1 and MtFTb2, was reduced in the late flowering $M t C D F d 1 \_1$ over expressing plants [12], while $M t F T a 1$ expression was precociously elevated in spring mutants that flower rapidly compared with wild type plants $[17,18]$.

The MtE1L gene is a Medicago homolog of the important Glycine max (soybean) photoperiodic flowering time regulator $E 1$, a legume-specific gene [4, 19]. A $M t E 1 L$ knock-out mutation caused a delay in flowering time in Medicago indicating a role in promoting flowering [19]. Functional analysis of the three Mt SUPPRESSOR OF OVEREXPRESSION OF CONSTANS1 (SOC1)-like genes identified a late flowering Mtsoc1a mutant, supporting a role for MtSOCla in promoting flowering [20]. Like MtSOC1a, MtSOC1b and MtSOC1c were photoperiodically regulated and were partly dependent on MtFTa1 for their expression [20, 21]. All three MtSOCs can partially complement the late flowering of the Atsoc1 mutant [21]. Two FRUITFULL-LIKE (FUL-like) genes, MtFULa and MtFULb, promoted flowering when over expressed in heterologous Arabidopsis and their expression was partly dependent on MtFTa1, indicating a likely role in flowering time control in Medicago [22]. On the other hand, MtVERNALISATION2 (VRN2) has a different function from that of Arabidopsis $V R N 2$, because $M t V R N 2$ represses MtFTa1 and flowering before vernalization [23]. In addition, while the two MtSHORT VEGETATIVE PHASE-LIKE (SVP-like) genes, $M t S V P 1$ and $M t S V P 2$, repressed flowering when overexpressed in Arabidopsis, over expression of MtSVP1 did not delay flowering in Medicago [24].

Medicago PHYTOCHROME A (PHYA) is a candidate photoperiodic flowering regulator whose function has not previously been reported. In Arabidopsis, this red/far red photoreceptor promotes flowering in LD by promoting $\mathrm{CO}$ protein accumulation in leaves. PHYA antagonizes the SUPPRESSOR of phyA-105/ CONSTITUTIVELY PHOTOMORPHOGENIC 1 (SPA/COP) complex, which degrades CO protein $[25,26]$. PHYA also plays a role in the entrainment of the Arabidopsis circadian clock and thus, the circadian-regulated diurnal expression of LD flowering time genes such as CO and GI [27, 28]. Despite the apparent absence of a Medicago CO-like function [11], a role for PHYA in promoting temperate legume photoperiodic flowering is strongly supported by previous classical genetic studies in pea. Pea phya loss of function mutants cause strong LD-specific delays to flowering and display architectural phenotypes similar to plants grown in short day (SD) conditions [29]. The important role of PSPHYA in promoting flowering is also shown by a dominant Psphya mutation that confers PHYA stability and causes early flowering [30]. 
Here, to further investigate photoperiodic flowering control in Medicago, we carried out a reverse genetic analysis of the PHYA gene, which exists as a single copy in the Medicago genome [31, 32]. We report the effect of two independent Medicago phya mutations on flowering time and plant architecture. We examine the expression of candidate flowering time and clock genes in the Mtphya-1 mutant in different environmental conditions and investigate genetic interactions by generating double mutant plants of Mtphya combined with either Mtfta1 or Mte1l.

\section{Results}

\section{Initial characterisation of MtPHYA}

MtPHYA (Medtr1g085160) is predicted to encode a 1124 aa protein containing the important domains that comprise the $\mathrm{N}$-terminal photosensory core module and the C-terminal regulatory region typical of PHYA-like proteins (Additional file 1: Figure S1) [27]. It is $79 \%$ identical to Arabidopsis PHYA, but shares highest homology with PHYA-like protein sequences from other temperate legumes including pea (95\%), red clover (95\%), chickpea (93\%) and lotus (89\%) (Additional file 1: Figure S1).

We analysed MtPHYA gene expression in different tissues and environments by qRT-PCR using gene-specific primers 2F and 2R (Fig. 2a, Additional file 2: Table S1). MtPHYA was detected in a wide range of tissues $2 \mathrm{~h}$ after dawn, including cotyledons, leaves, apical buds, open flowers and roots (Fig. 1a) and was slightly more abundant in SD than in LD photoperiods (Fig. 1b). Analysis of MtPHYA expression in a developmental timecourse in LD in leaves and shoot apices indicated that it did not change significantly in leaves, except for a 2-fold increase prior to flowering, but its expression declined after flowering (Fig. 1c). In contrast, in shoot apices, there was a steady increase in expression through development and it continued to rise after flowering (Fig. 1d).

Medicago flowering is promoted by prolonged winter cold (vernalization, V) followed by long-day (LD) photoperiods [10]. To assess if vernalization has a direct effect on MtPHYA transcript, we analysed its expression in a vernalized seedling long-day (VsLD) time course. Seeds were germinated, grown in LD, then 14-d-old seedlings were vernalized at $4{ }^{\circ} \mathrm{C}$ and afterwards returned to warm LD. MtPHYA was expressed in leaves and apices of seedlings before, during and after vernalization at similar levels, although at a significantly lower level than in germinated seeds (Fig. 1e-f). This indicates that MtPHYA expression is not directly regulated by cold. The high abundance of MtPHYA in etiolated germinated seeds (Fig. 1e), is consistent with other plants including pea where PHYA transcript accumulates to a much higher level in the dark than in the light $[29,30,33]$.
Two independent Mtphya mutant lines have reduced sensitivity to far-red light compared with wild type R108 seedlings

To investigate the function of MtPHYA in Medicago plant development, we analysed two independent Tnt1 retrotransposon-tagged R108 mutant lines, Mtphya-1 (NF1583) and Mtphya-2 (NF3601), which have insertions facing in opposite directions in the $5^{\prime}$ UTR of MtPHYA (Fig. 2a). To analyse if the Tnt1 insertions affected the full-length transcript of MtPHYA, we used primers 3F and 3R (Fig. 2a, Additional file 2: Table S1) to amplify cDNA fragments from wild type R108 and the two mutant lines. The primers amplified cDNA fragments from both R108 and Mtphya-1, but none from Mtphya-2 indicating that the latter was a knockout mutant (Fig. 2b). Direct sequencing of the PCR product from R108 indicated that the intron upstream of the ATG in R108 was spliced out using the $5^{\prime}$ splice donor site at position - 522 to generate a 3533 bp cDNA. However, sequencing of the PCR product from Mtphya-1 indicated alternative splicing of this upstream intron, which utilized a $5^{\prime}$ splice donor site at position -611 . This led to splicing out of the Tnt1 insertion and adjacent $89 \mathrm{bp}$ in the 5'UTR of Mtphya-1, which resulted in amplification of a slightly shorter cDNA (3444 bp). As measured by qRT-PCR using primers $2 \mathrm{~F}$ and $2 \mathrm{R}$, Mtphya-1 and Mtphya-2 had a statistically significant 3 -fold and $\sim 11$-fold reduction, respectively, in $M t P H Y A$ gene expression, compared with wild-type R108 plants (Figs. 2c, 4a).

Because PHYA has a well-documented role in regulation of seedling photomorphogenesis [34, 35], we then analysed seedling de-etiolation responses of the two Mtphya mutant lines and wild-type R108 (Figs. 2d-e, 4b). Seeds were germinated in the dark overnight and then grown for three days either under far-red (FR) light, in the dark (D) or in white light (WL). Under continuous FR light, both the Mtphya mutants had longer hypocotyls with unexpanded cotyledons, compared to wild type R108 seedlings with short hypocotyls and expanded, green cotyledons (photographs shown for Mtphya-1, Fig. 2d). In contrast, in WL, the Mtphya mutants had short hypocotyls that were only slightly longer than wild-type R108 hypocotyls (photographs shown for Mtphya-1, Fig. 2d). In dark, the hypocotyls of both mutants were very long like dark-grown, wild-type R108 seedlings (photographs shown for Mtphya-1, Fig. 2d). When the hypocotyl lengths were plotted as a ratio of light to dark grown, the WL/Dark hypocotyl length ratios were low in the wild-type and both the Mtphya mutants (Figs. 2e, 4b). However, the FR/Dark hypocotyl length ratio in the Mtphya mutants was much higher than in R108 (Figs. 2e, 4b). These results indicate that the Mtphya mutants had reduced sensitivity to far-red light. 
A

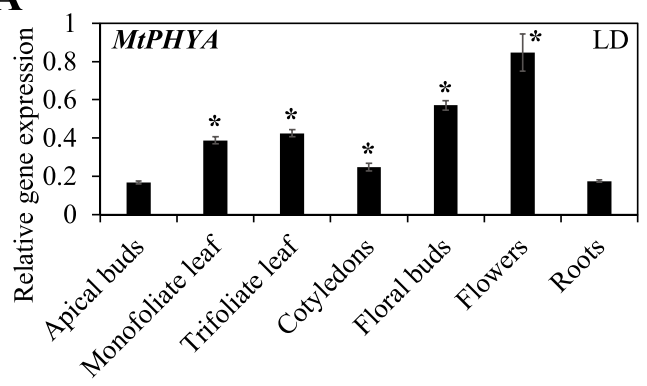

C

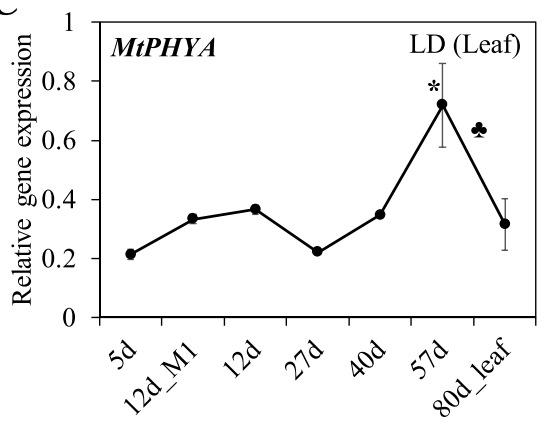

E

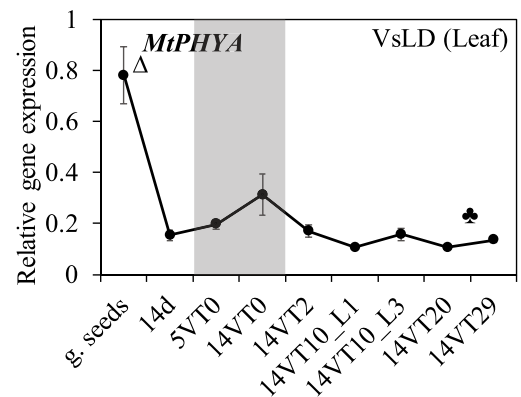

B

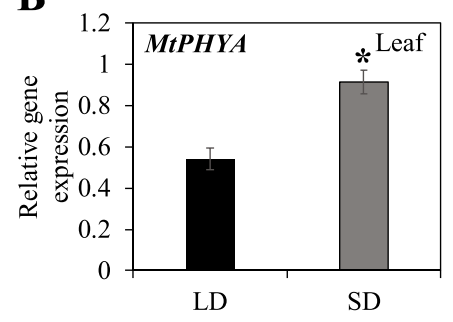

D

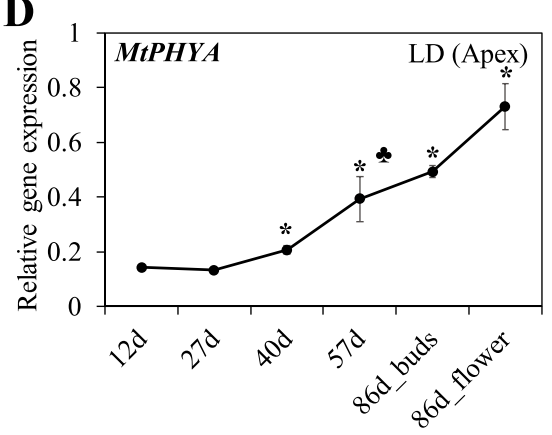

F

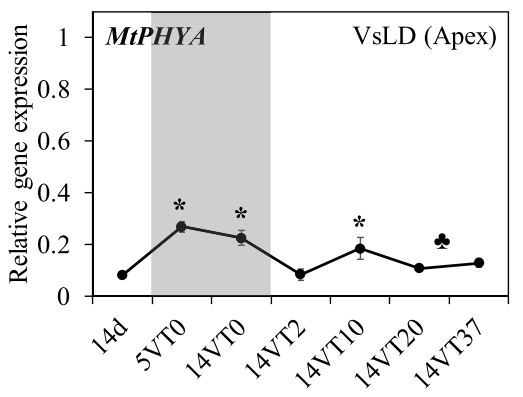

Fig. 1 MtPHYA is expressed broadly and throughout development in Medicago wild type R108 plants. a Relative expression of MtPHYA in tissues of plants grown in long-day (LD) photoperiods. The cotyledons were from 5-d-old seedlings; monofoliate and trifoliate leaves, apical buds and roots from 12-d-old plants; and floral buds (young) and flowers from 86-d-old flowering plants. b Relative transcript abundance of MtPHYA in fully-expanded trifoliate leaves of 14-d-old seedlings grown under LD and short-day (SD) photoperiods. c-d Relative gene expression levels of MTPHYA in leaves (c) and uppermost apical buds, flower buds or open flowers (d) in days after planting in LD. The in (c-d) indicate that the plants flowered with floral buds first visible at 69 days. Cotyledons were harvested at 5 days while monofoliate leaves (M1) or trifoliate leaves were harvested at the remaining time points. e-f MtPHYA expression in germinated seeds (g. seeds) and in leaves (e) and uppermost shoot apices ( $\mathbf{f}$ ) of plants before vernalization (14d), during vernalization at $4^{\circ} \mathrm{C}$ (shaded) with sampling done after 5 days $(5 \mathrm{VT} 0=5$ days in the cold, 0 day warm) and 14 days (14VTO), and after the plants were transferred to warm LD conditions (VsLD) $(14 \mathrm{VT2}=2$ days in the warm after 14 days of vernalization). The in (e-f) indicate that the plants flowered with floral buds first visible at 52 days after planting. The tissues in (a-f) were harvested two hours after dawn (ZT2). Gene expression was determined using qRT-PCR with primers 2F and 2R (Additional file 2: Table S1) and is shown as the mean \pm se of three biological replicates, normalized to Medicago PP2A and relative to the highest value in (a and $\mathbf{b})$. For $\mathbf{c}-\mathbf{d}$ and $\mathbf{e}-$ $\mathbf{f}$ the data is shown relative to the highest value over both tissues. The ${ }^{*}$ indicates significantly different expression from the first data point in (a$\mathbf{d}, \mathbf{f})$ while the $\Delta$ in (e) indicates that the expression in germinated seeds is significantly different from the rest of the data points [multiple pairwise comparisons adjusted for false discovery rate (FDR); $a=0.05]$

\section{Mtphya mutants flower later than wild type R108 particularly in LD and VLD photoperiods}

To investigate if the Mtphya mutations affect flowering time, we characterized the mutant plants for their flowering time phenotypes compared with wild type R108 plants. First, under vernalized long day photoperiod (VLD) conditions, both Mtphya-1 and Mtphya-2 mutant plants were late flowering compared with R108 (Figs. 2f$\mathrm{g}, 4 \mathrm{c}-\mathrm{d})$. We then crossed homozygous Mtphya-1 mutants with wild-type R108 control plants and analysed the genotype and flowering time of the F1 plants and the segregating F2 population in VLD (Fig. 2f-h). The F1 progeny had a weak intermediate phenotype, flowering slightly later than wild-type R108, but much earlier than 

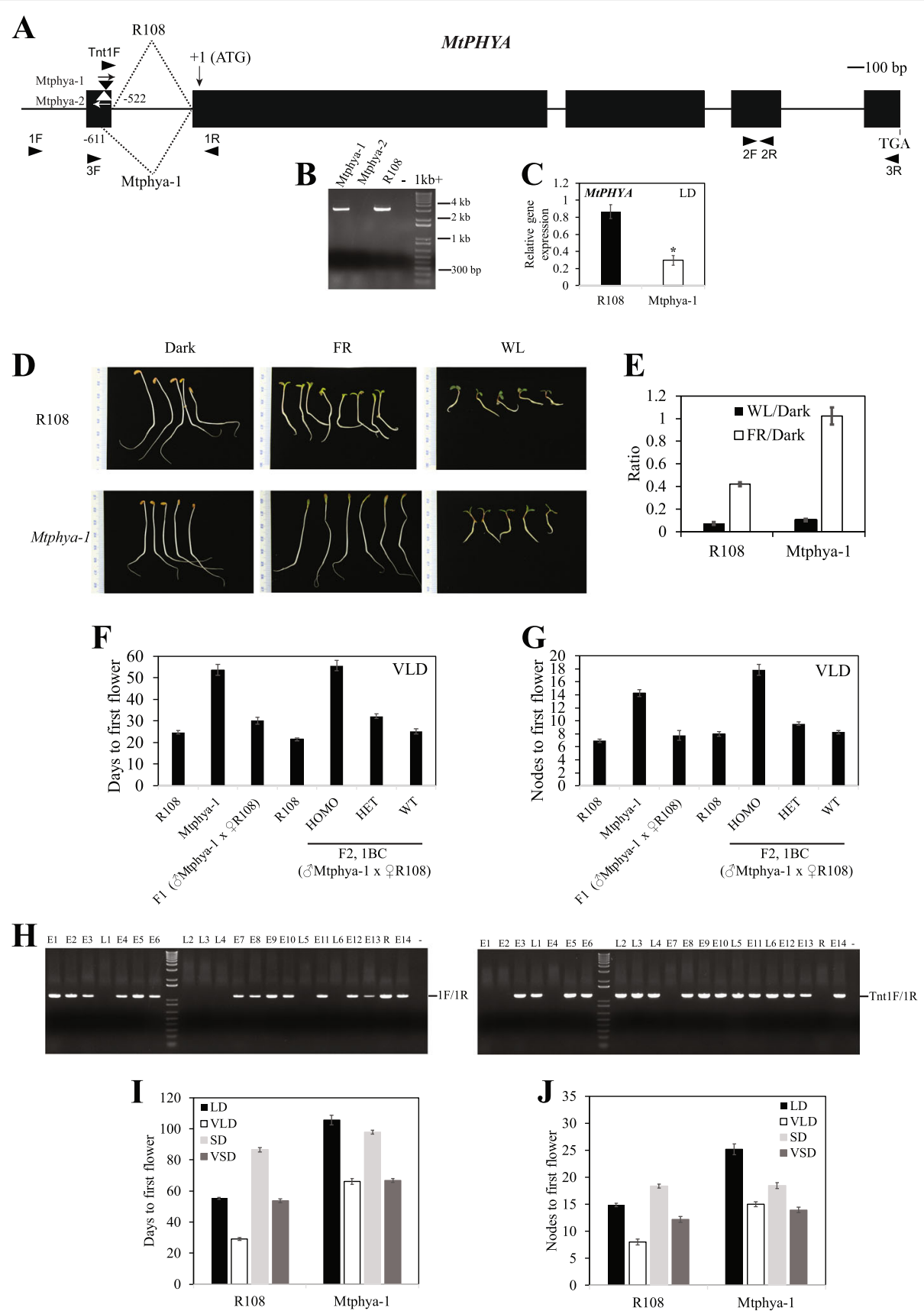

Fig. 2 The Mtphya-1 mutant has reduced sensitivity to far-red light and flowers later than wild type R108 particularly in LD and VLD photoperiods. a MtPHYA with Tnt1 insertions at - 536 bp and -556 bp upstream of the ATG in the Mtphya-1 (black triangle) and Mtphya-2 (white triangle) mutants, respectively. Exons are black boxes and introns are thin lines. Arrows indicate orientation of Tnt insertions. Dotted lines indicate splice sites used in Mtphya-1 compared with R108. $\mathbf{b}$ cDNA fragments amplified by primers 3F and 3R. c MtPHYA expression in 14-d-old seedlings in LD, $2 \mathrm{~h}$ after dawn, using qRT-PCR with primers $2 \mathrm{~F}$ and $2 \mathrm{R}$. Mean \pm SE three biological replicates, normalized to Medicago PP2A and relative to the highest value. ${ }^{*}$ significantly different expression from R108 using one-way analysis of variance (ANOVA) test between the means ( $(a=0.05)$. $\mathbf{d}$-e Seedlings in white light (WL), far-red, FR and dark (d) and ratio of hypocotyl lengths of 3-d-old seedlings to dark-grown (e). Mean \pm (t.SE) $(0.05), n=9$. (f-g) Flowering time in vernalized LD (MLD) scored as days to flowering $(\mathbf{f})$ or the number of nodes on the primary axis at flowering $(\mathbf{g})$ of the F1 progeny $(n=8)$ from Mtphya-1 crossed to R108, Mtphya-1 (self-cross) $(n=21)$, R108 ( $n=18)$ and segregating F2 progeny ( $n=217$ : Mtphya-1 Tnt1 homozygotes, $n=50$; heterozygotes, $n=114$; wild-type segregants, $n=53)$ with R108 $(n=25)$. Data are mean \pm (t.SE) (0.05). (h) PCR genotyping fragments from segregating F2 plants in (F-G). Plants were scored as early (e) (like R108) or late (I) flowering relative to R108. 1F and 1R used for wild-type band and 1R and Tnt1F for Tnt1. i-j Graphs showing the flowering time in different conditions of Mtphya-1 mutants (no backcross) and R108 scored in days (i) or nodes to first flower (j). Mean \pm (t.SE) (0.05) is presented $(n=9-16)$ 
Mtphya-1 mutants. In the segregating F2 population of 217 plants, $\sim$ one quarter $(n=50)$ were Mtphya-1 Tnt 1 homozygotes and late flowering, $\sim$ half $(n=114)$ were heterozygotes and flowered slightly later than wild-type R108 and wild-type segregants, and $\sim$ a quarter $(n=53)$ were wild-type segregants and flowered like wild-type R108. Thus, the Tnt1 insertion in Mtphya-1 was tightly linked to the late flowering locus (within $\leq 1 \mathrm{cM}$ ).

The Tnt1 insertion in Mtphya-2 also showed 100\% cosegregation with the late flowering phenotype. The pattern of inheritance in Mtphya-2 was analysed by characterising the flowering time of a segregating population from heterozygous, self-crossed parents in VLD. Out of 45 plants, about one quarter $(\mathrm{n}=11)$ were homozygotes and all were late flowering, and $\sim$ one quarter $(n=8)$ were wild-type segregants and early flowering like wild-type R108. The remaining plants $(n=26)$ were heterozygotes, which showed semi-dominance as observed for Mtphya-1, because they displayed an intermediate late flowering time phenotype (Fig. 4c-d).

To further investigate the role of MtPHYA in regulation of flowering, Mtphya-1 plants were grown under different photoperiodic conditions, with or without, vernalization treatment of germinated seeds (Fig. 2i-j). As expected, the R108 wild-type plants exhibited a strong response to photoperiod and vernalization, flowering most rapidly in vernalized long day (VLD) but flowered the latest under non-vernalized short day (SD) conditions. However, the Mtphya-1 mutants were strikingly impaired in their ability to respond to LD compared with wild type plants. Mtphya-1 mutants were delayed in flowering in both LD and VLD compared to R108, but flowered at a similar time to wild type R108 in VSD and SD. The mutants exhibited a late flowering day-neutral phenotype, particularly in vernalized conditions, as they flowered at a similar time in VLD and VSD. The Mtphya-1 mutants retained the ability to respond to vernalization because they flowered earlier in VLD than LD, and similarly, the VSD-grown mutants were earlier than the SD-grown plants. However, the response of Mtphya-1 to vernalization was slightly weaker compared with R108.

\section{Mtphya mutants have a very short primary axis in LD and VLD photoperiods compared with wild type}

In addition to displaying delayed flowering, both the Mtphya mutant plants were more compact than wild type R108, with a strikingly short primary axis (see photographs of Mtphya-1 mutant in Fig. 3a-b). This phenotype was more pronounced in LD photoperiods (both LD and VLD) than in SD or VSD conditions in the Mtphya-1 mutant compared with R108 (Fig. 3a-d). Measurements of primary axis lengths, taken at different stages of development, indicated that both the Mtphya mutants exhibited the short axis phenotype compared to R108, prior to and after flowering (Fig. 3e-f, Fig. 4g). To analyse the inheritance of the short primary axis phenotype, we also measured primary axis length in VLD in the population segregating for the Mtphya-2 mutation (Fig. 4f), previously analysed for flowering time (Fig. 4cd). The short axis phenotype was only observed in the Mtphya-2 homozygous segregants (Fig. 4f). Thus, there was co-segregation between the short primary axis phenotype and the late flowering phenotype (Fig. 4c-d). These results indicate that the late flowering time defect and the short primary axis phenotype are both caused by mutations in the MtPHYA gene.

\section{The delayed flowering of Mtphya-1 in LD and VLD is associated with a decrease in expression of LD-induced MtFTs, MtFULs and MtE1L}

Next, we analysed the molecular basis of the altered long day photoperiod flowering time response of Mtphya-1 mutants. To do this, we analysed the expression of candidate Medicago circadian clock and flowering-time genes in leaves of the Mtphya-1 mutant and wild type plants in LD and SD (Fig. 5) and in VLD (Fig. 6) two hours after dawn (ZT2) using qRT-PCR.

The expression level of GI was similar in LD and SD in wild type R108 and was reduced by $\sim 3$-fold in the Mtphya-1 mutants compared with R108 in LD, but not in SD (Fig. 5). GI was reduced by $\sim 2$-fold in the mutant in VLD (Fig. 6). TOC1a level increased by $\sim 4$-fold in the Mtphya-1 mutant in SD compared with R108, but was unchanged in LD (Fig. 5) at ZT2. However, it was reduced by $\sim 2$-fold in the mutant in VLD compared with R108 control (Fig. 6a). The other Medicago clock-related genes homologous to ELF3, ELF4, LUXa, LUXb and $L H Y$ were analysed only in VLD, but were not changed compared with wild type R108 (Fig. 6a). The expression of a candidate flowering-time gene, FKF1-like, was also not changed in the mutant compared with wild type in VLD (Fig. 6a).

The expression of the three LD-induced $M t F T$ genes, MtFTa1, MtFTb1 and MtFTb2 [16] were strikingly reduced in the Mtphya-1 mutant. MtFTb1 and $M t F T b 2$ were highly expressed in LD but undetected under SD in R108, consistent with previous findings [16]. However, both genes were strongly decreased by $\sim 16$ - and $\sim 19$-fold, respectively, in the Mtphya-1 mutant compared with wild type in LD (Fig. 5). In VLD, MtFTa1, MtFTb1 and MtFTb2 were strongly decreased by $\sim 39-, 24-$ and 11 -fold, respectively in the Mtphya-1 mutants compared with wild-type R108 (Fig. 6b). In contrast, the fourth FT-like gene tested, $M t F T a 2$, was weakly reduced by $\sim 2$-fold in the mutant in VLD (Fig. 6b). 

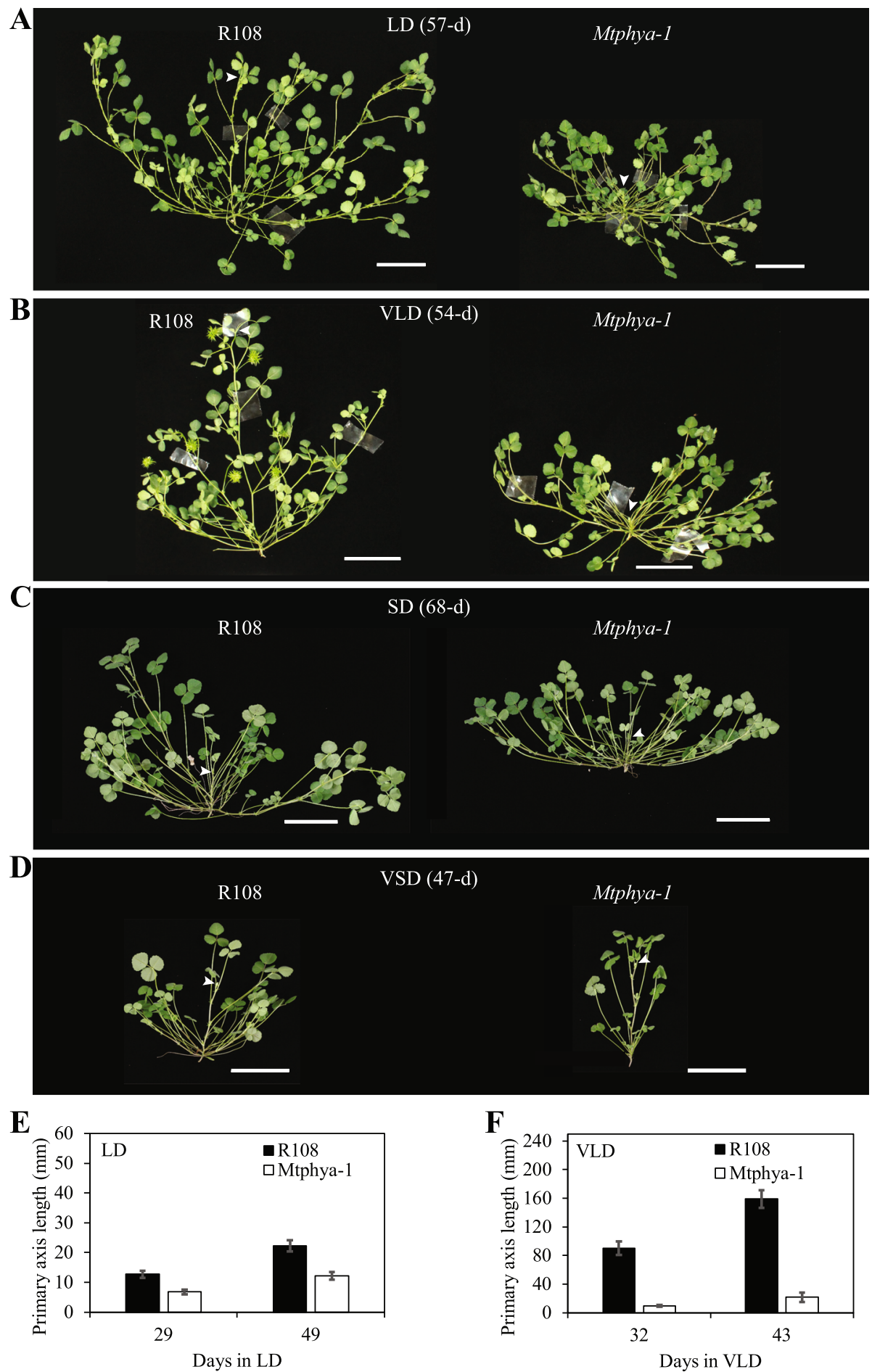

Fig. 3 The Mtphya-1 mutant has a very short primary axis in LD and VLD compared to R108. a-d Photographs of wild type R108 and Mtphya-1 (non-backcross) mutants in LD (a), VLD (b), SD (c) and VSD (d). Arrowheads indicate the primary axis. Scale bar $=5 \mathrm{~cm}$. e-f Graphs showing the lengths of the primary axis of wild-type R108 and Mtphya-1 mutants at different days of growth in LD (e) and VLD (f). The LD Mtphya-1 plants were F3 homozygous mutants after a backcross to R108 while the VLD Mtphya-1 plants were F2 homozygous mutants after two backcrosses to R108. The data are shown as the mean \pm (t.SE) (0.05) $(n=5-10)$ 


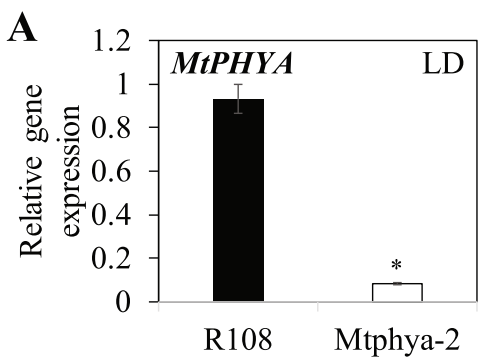

C

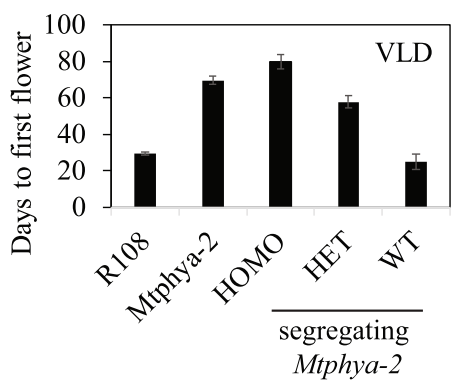

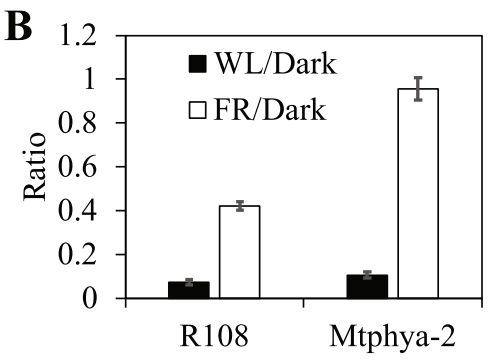

D

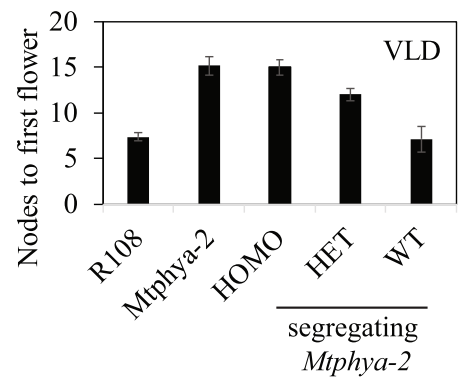

$\mathbf{E}$
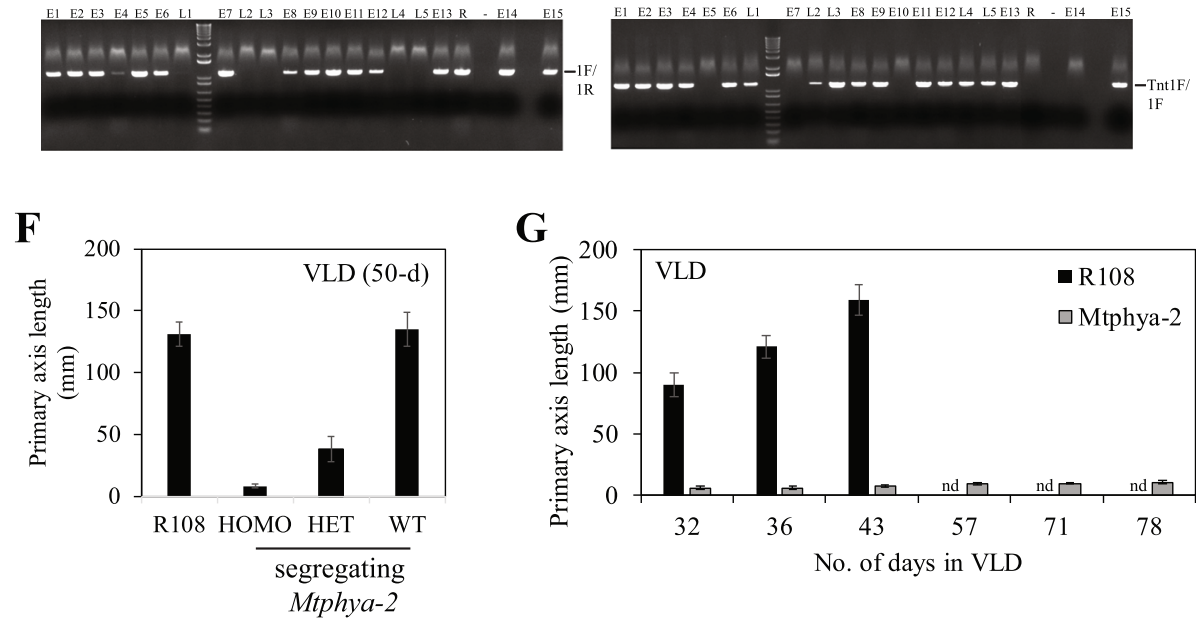

G

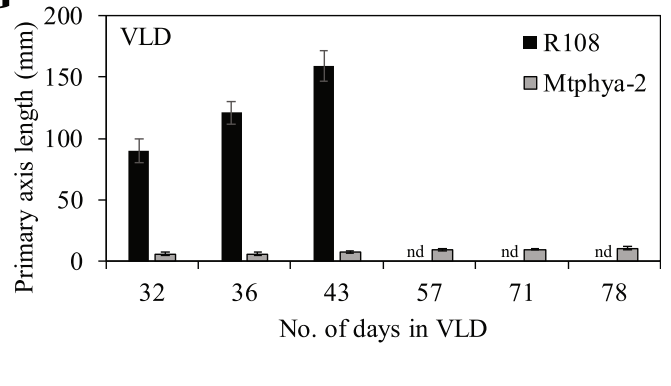

Fig. 4 The Mtphya-2 mutant has reduced sensitivity to far-red light, is late flowering and has a very short primary axis throughout development in VLD compared to R108. a Relative expression of MtPHYA in 25-d-old plants in LD $2 \mathrm{~h}$ after dawn using qRT-PCR with 2F and $2 \mathrm{R}$ primers. Data are the mean \pm SE of three biological replicates, normalized to Medicago PP2A and relative to the highest value. The * indicates significantly different expression from wild type R108 using one-way ANOVA test between the means $(a=0.05)$. $\mathbf{b}$ Ratio of hypocotyl lengths of 3-d-old seedlings of R108 and Mtphya-2 in different light conditions (white light, WL; far-red, FR) to dark-grown. Data are mean \pm (t.SE) (0.05), $n=9$. c-d Graphs showing VLD flowering time in days (c) or the number of nodes on the primary axis at flowering (d) of a segregating population of Mtphya-2 heterozygous plants (self-crossed) ( $n=45$ : Mtphya-2 homozygotes, $n=11$; heterozygotes, $n=26$; wild-type $n=8$ ) compared with Mtphya-2 mutants (self-crossed) $(n=6)$ and R108 plants $(n=10)$. Data are the mean \pm (t.SE) $(0.05)$. e PCR genotyping fragments from segregating Mtphya-2 plants in (c-d). Plants scored as early (E) (like R108) or late (L) flowering relative to R108. Genotyping primers $1 \mathrm{~F}$ and 1R (Fig. 2A) were used for wild-type band and primers $1 \mathrm{~F}$ and Tnt1F for Tnt1 insertion. f Primary axis lengths in VLD of segregating Mtphya-2 plants in (c-e) compared with R108. Measurements are the mean \pm (t.SE) (0.05) $(n=8-26)$. g Lengths of primary axis of R108 and Mtphya-2 over time in VLD. Mtphya-2 mutants were homozygous F2 plants after backcrossing to R108. Data are mean \pm (t.SE) (0.05) ( $n=8-10)$. R108 plants flowered at $23-26$ d while Mtphya-2 mutants at $\sim 65-71$ d in VLD. nd, not done

MtFULb was previously shown to be under photoperiodic control because it was expressed at higher levels in LD than in SD [17]. A similar result was observed here (Fig. 5). MtFULa was also photoperiodically regulated with strongly elevated expression in the leaves of wild type R108 under LD compared with SD (Fig. 5). The Mtphya-1 mutation had a particularly strong effect on the expression of MtFULa because it was decreased by $\sim 15$-fold, while MtFULb was reduced by $\sim 3.5$-fold in the Mtphya-1 mutant 

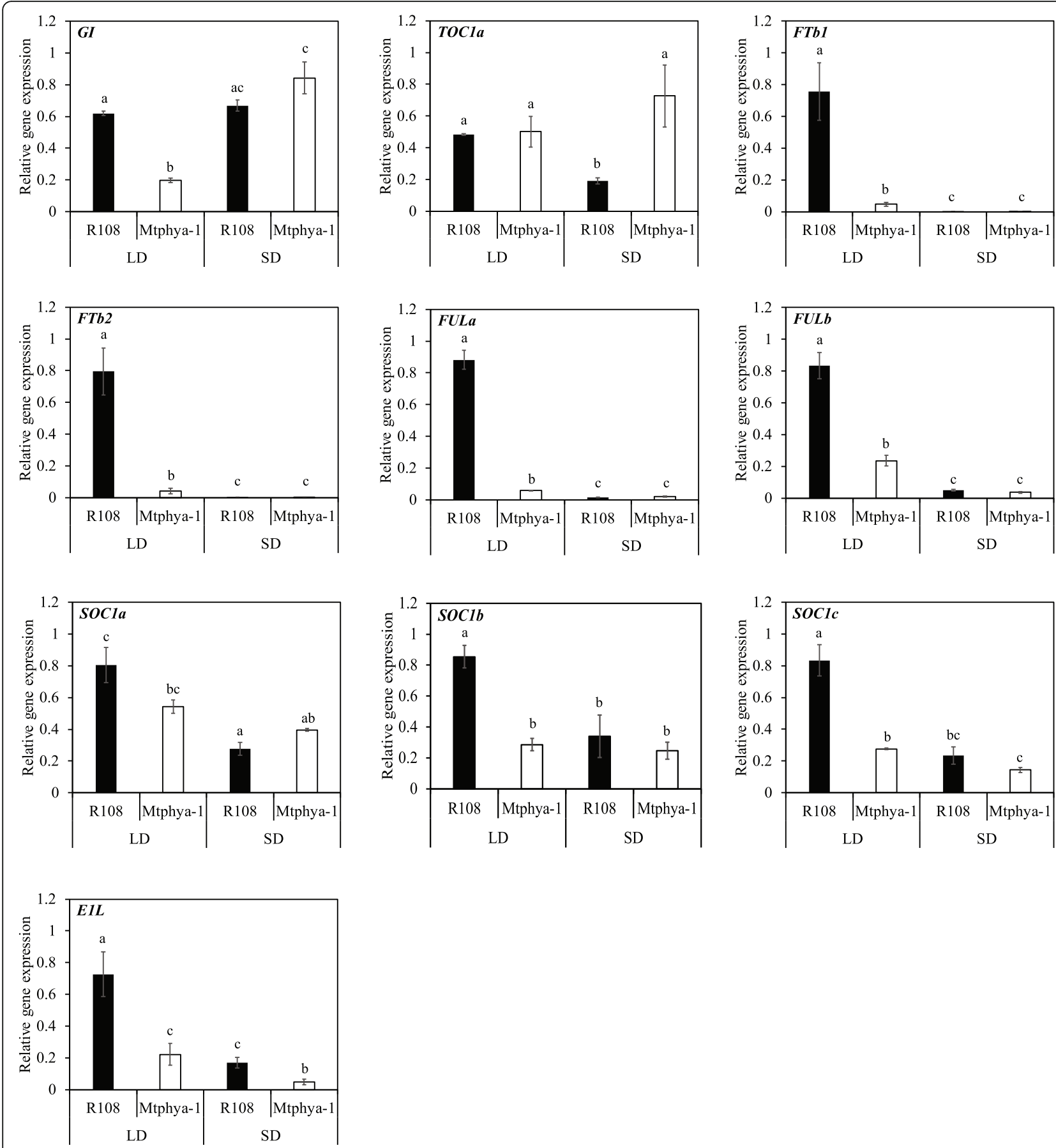

Fig. 5 Gene expression of candidate photoperiod and flowering time genes in the Mtphya-1 mutant and R108 in LD and SD. Relative transcript abundance was measured in the fully expanded trifoliate leaves of 14-d-old wild-type R108 and Mtphya-1 homozygous seedlings. Relative gene expression was measured by qRT-PCR with normalization to PP2A. Data are the mean \pm se of three biological replicates and relative to the highest value. Tissues were harvested $2 \mathrm{~h}$ after dawn. Different letters $(\mathbf{a}, \mathbf{b}, \mathbf{c})$ indicate significant differences, while the same letter(s) on a bar indicate no significant differences using the two-way ANOVA test [multiple pairwise comparisons adjusted for false discovery rate (FDR); $a=0.05$ ]. Tissues were harvested $2 \mathrm{~h}$ after dawn

compared with wild type in LD. Similarly, in VLD, MtFULa was reduced by $\sim 8.3$-fold and MtFULb by $\sim 3$-fold in the Mtphya-1 mutant compared with wild type R108 (Fig. 6c).
The three MtSOC1 genes were expressed at higher levels in LD than in SD in R108 as shown in previous work [20, 21]. However, the expression of MtSOC1a was not significantly changed in the leaves of the Mtphya-1 


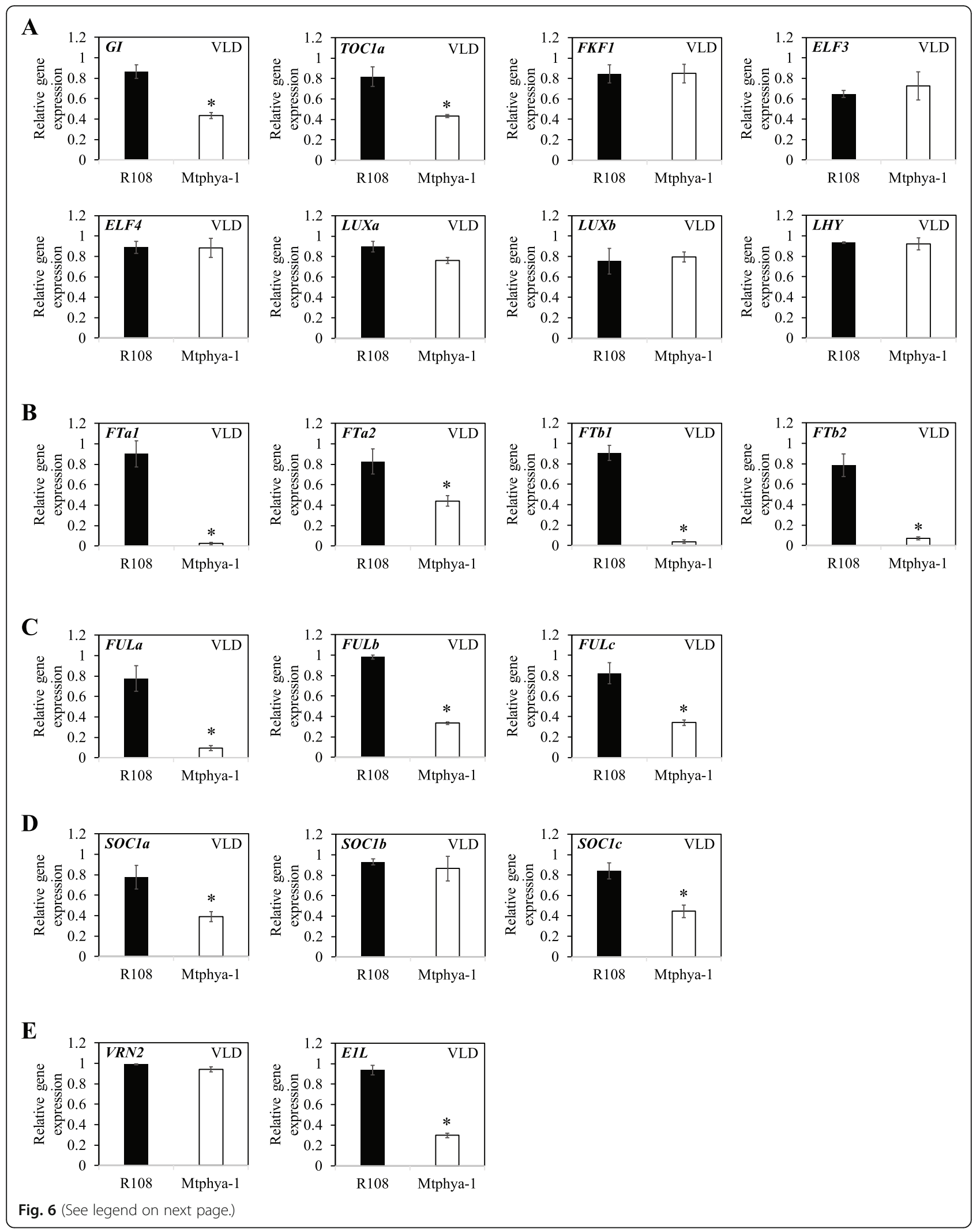


(See figure on previous page.)

Fig. 6 Gene expression of candidate circadian and flowering time genes in the Mtphya-1 mutant and R108 in VLD. Relative transcript abundance was measured in the fully expanded trifoliate leaves of 14-d-old wild-type R108 and Mtphya-1 homozygous seedlings grown in vernalised longday (VLD) photoperiods. Relative gene expression was measured by qRT-PCR with normalization to PP2A. Data are the mean \pm t.se of three biological replicates and relative to the highest value. The * indicates significantly different expression from wild type R108 using one-way ANOVA test between the means $(a=0.05)$. Tissues were harvested $2 \mathrm{~h}$ after dawn

mutants compared with R108 in either LD or SD conditions. MtSOC1b and MtSOC1c decreased by $\sim 3$-fold in the mutant in LD but were unchanged in SD (Fig. 5). In VLD, the Mtphya-1 mutants showed a weak decrease in the expression of MtSOC1a and MtSOC1c ( 2-fold) but not in MtSOC1b compared with R108 (Fig. 6d).

We observed that $M t E 1 L$ is $~ 3.5$ fold more abundant in LD than in SD in R108, indicating that it is also photoperiodically controlled (Fig. 5). The same pattern was observed for the expression of MtE1L in the Mtphya-1 mutant. However, the mutant plants showed a reduction in $M t E 1 L$ transcript level by 3-fold in LD and SD compared with R108 control and a similar pattern was observed under VLD (Fig. 6e).

There was no significant change in the transcript level of MtVRN2 [23] in the leaves of Mtphya-1 mutants compared with wild type (Fig. 6e).

\section{Mtphya-1 Mte1/ double mutants flower at the same time as the Mtphya-1 mutant in VLD}

Since $M t E 1 L$ expression is under LD photoperiodic control and its level decreased in the Mtphya-1 mutant, we tested genetically if $M t E 1 L$ and $M t P H Y A$ promoted flowering via a common pathway. The Mtell mutant line (NF16583) with a Tnt1 insertion in the MtE1L coding region was obtained [19]. The Mte1l single mutants were moderately delayed in flowering compared with wildtype R108 controls in VLD (Fig. 7). We then crossed Mtphya-1 with Mte1l and genotyped and scored the flowering time of the segregating F2 population from the cross. Three out of 59 ( 1/16) F2 plants were Mtphya-1 Mte1l double mutants. These plants flowered late in VLD at a similar time to the single Mtphya-1 mutants (Fig. 7). Therefore, no additive effect on flowering time was seen indicating that Mte1l and Mtphya-1 were likely to be in the same pathway. The other Mtphya-1 homozygous plants, either wild type or heterozygous at $M t E 1 L$, were also similarly delayed in flowering comparable to Mtphya-1 mutant (Fig. 7). However, only a very weak effect on flowering time was seen in the one MtPHYA wild type/Mtell homozygous F2 plant obtained, consistent with the mild effect of Mte1l on flowering in VLD (Fig. 7).

\section{Mtphya-1 fta1 double mutants flower slightly later than either of the single mutants in VLD}

Plants with a knockout mutation at MtFTa1 are late flowering with short primary axes and prostrate architecture [16], similar to the Mtphya mutants. In addition, MtFTa1 expression is strongly reduced in the Mtphya-1
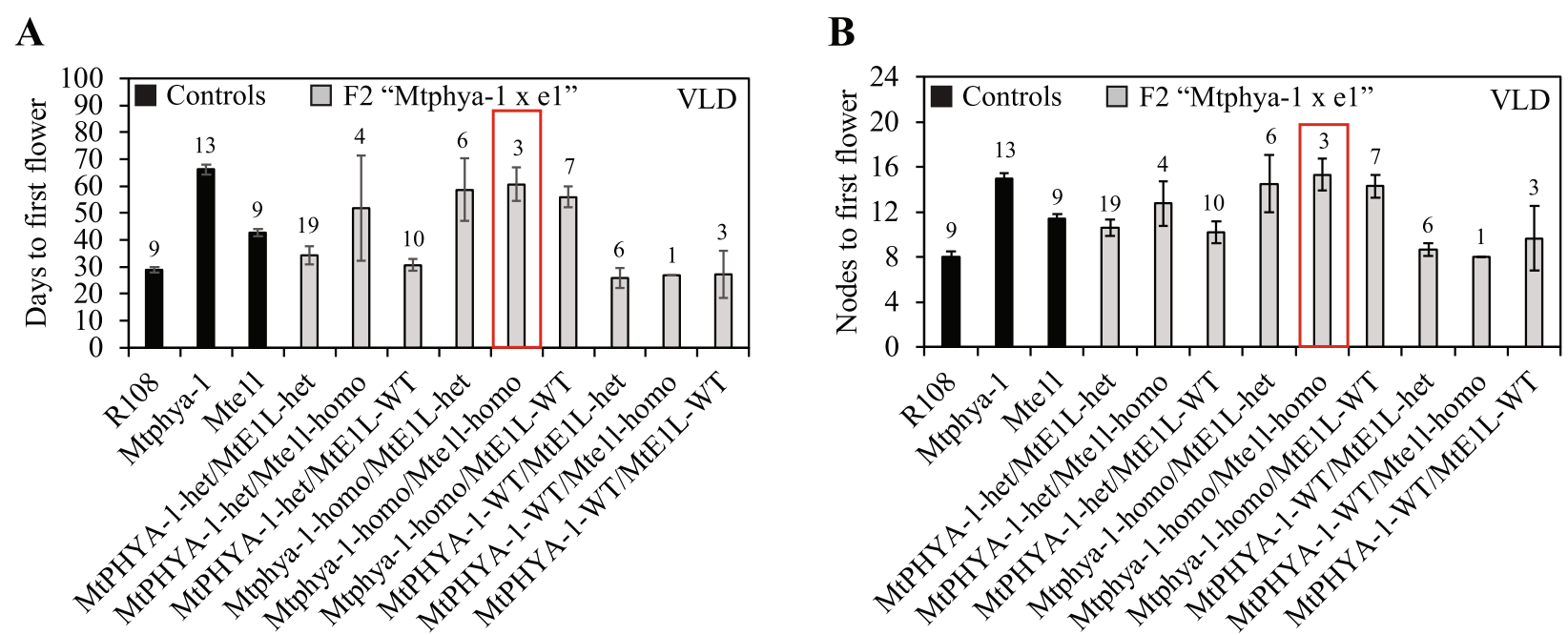

Fig. 7 Mtphya-1 ell double mutants flower at the same time as the Mtphya-1 single mutant in VLD. Average flowering time of controls and the F2 population of Mtphya-1 x Mte1/ in VLD. Flowering time was scored as either days (a) or nodes (b) to first flower and are shown as the mean \pm (t.SE) (0.05). The numbers of plants scored of each genotype are shown above the bars. The box indicates the double Mtphya-1 Mte1/ mutant plants 
mutant in VLD. Thus, to test if MtFTa1 and MtPHYA promote flowering in the same flowering time pathway, we crossed Mtphya-1 and ftal mutants to generate a double mutant. In our initial experiment, we obtained six F2 plants, but genotyping indicated that none were double mutants. Therefore, a F2 Mtphya-1-homozygous/ FTa1-heterozygous plant was selected, self-crossed, and its F3 progeny were genotyped and scored.

We identified 9 Mtphya-1 ftal double mutants out of 48 F3 plants. They flowered later (by $\sim 2$ weeks and with 3-4 more nodes) than the Mtphya-1-homozygous F3 plants (Fig. 8a-b). They also flowered later than Mtfta1 single mutants grown as controls (Fig. 8a-b). This indicates that the mutations at both loci caused a weak additive effect compared with the single mutants in VLD. In addition, the 24 Mtphya-1 homozygous/FTa1 heterozygous plants flowered slightly later than the Mtphya-1 homozygous/FTa1 wild-type plants (Fig. 8a-b). This suggests that fta1 and Mtphya-1 largely affect flowering in the same pathway, but also possibly under different pathways. Apart from the delay in flowering time, mutations at both loci also caused a weak additive effect on reduction of the primary axis length (Fig. 8c), with a slight reduction in the length of the longest secondary axis (Fig. 8d).

\section{Discussion}

PHYA photoreceptors regulate photoperiodic flowering in several plants including Arabidopsis, the temperate
A

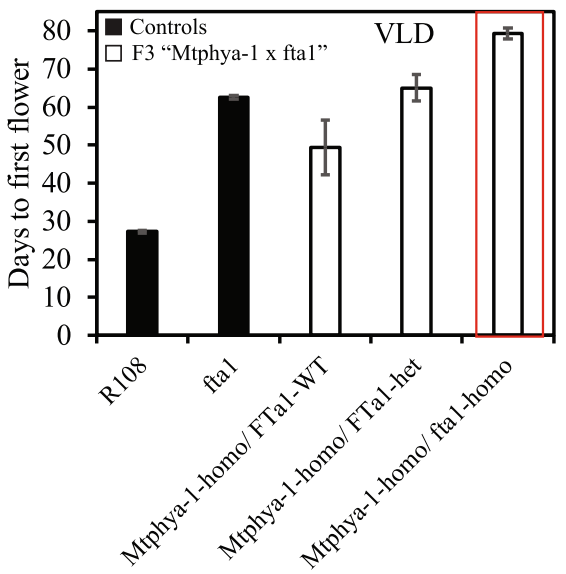

C

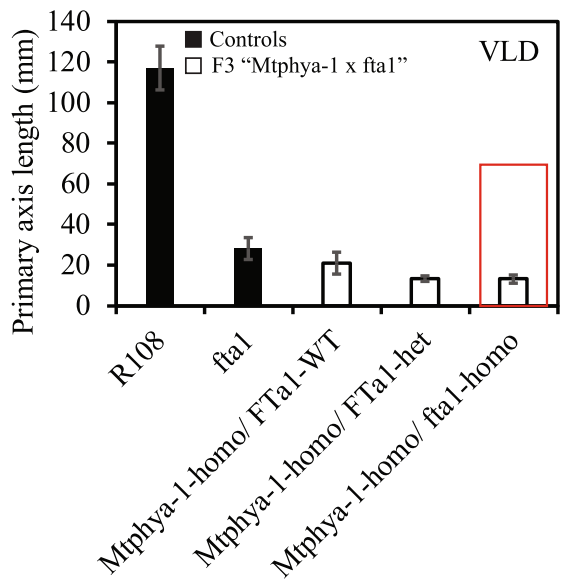

B

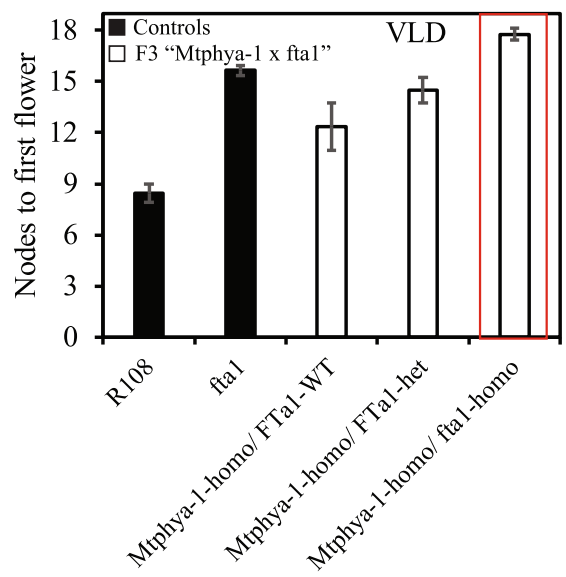

D

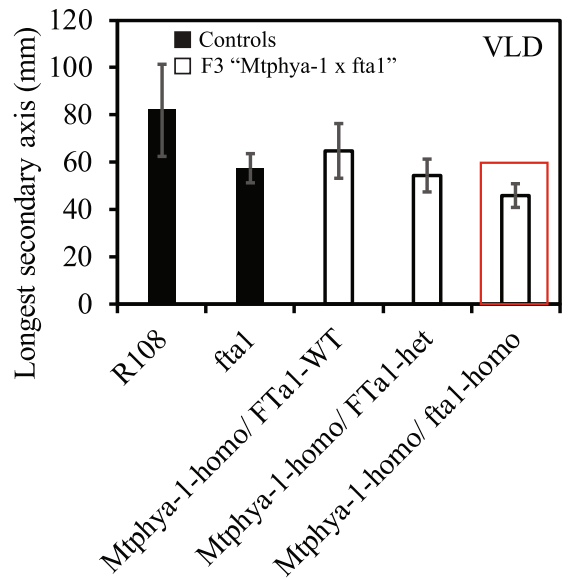

Fig. 8 Mtphya-1 ftal double mutants show weakly additive effects on flowering time and primary axis length in VLD. a-b Double Mtphya-1 fta 1 mutants were generated by crossing the single mutants. Average flowering time in VLD of controls (wild-type R108, $n=9 ;$ fta1 mutants, $n=14$ ) and F3 progeny of a Mtphya-1 homozygous/FTa1 heterozygous plant from Mtphya-1 x fta1 ( $n=48$ : Mtphya-1 homozygous/FTa1 wild-type, $n=15$; Mtphya-1 homozygous/FTa1 heterozygous, $n=24 ;$ Mtphya-1 homozygous/fta1 homozygous, $n=9$ ). Flowering time was scored as either days (A) or nodes (B) to first flower and are shown as the mean \pm (t.SE) (0.05). The box indicates the double Mtphya-1 fta1 mutant plants. c-d Average lengths of the primary (c) and longest secondary axis (d) of the plants in (a-b). The measurements were taken at 38-d-old in VLD and shown as the mean \pm (t.SE) (0.05) $(n=9-24)$ 
legume pea and the tropical legumes soybean and common bean [28, 29, 36-38]. Here, we demonstrate using reverse genetics that MtPHYA has a major role in LD photoperiodic regulation in Medicago. It is required for the promotion of Medicago flowering by LD and VLD, but has little effect under SD and VSD. The Mtphya-1 mutant had a late-flowering day-neutral phenotype compared with wild type R108 plants, particularly under vernalized conditions because the mutants flowered at the same time under VLD and VSD.

Mtphya mutants also displayed a very short primary axis early on development in LD and VLD and this was maintained even after flowering, in contrast to wild type plants. Thus, our work implicates MtPHYA in promoting primary axis elongation in Medicago in LD and VLD. Although the role of PHYA in seedling deetiolation under continuous FR light is well characterized in many plant systems $[29,34,35,39,40]$, there are exceptions. For example, in some legume species where the $P H Y A$ gene has been duplicated, genes including the PHYA-like, PHOTOPERIOD (PPD) in common bean [38] and the $E 3$ gene in soybean [36] do not perform this role. In Medicago, our results indicate that MtPHYA regulates Medicago seedling de-etiolation under FR light.

The role of PHYA photoreceptors in regulating flowering time through their effect on FT expression has been shown in tropical legume plants such as soybean [41] and common bean [38] but not reported previously for temperate legumes. Analysis of Medicago ftal mutants [16] and Mtphya mutants indicated similarities in their phenotype, including late flowering and prostrate plant architectures with very short primary axes. Moreover, the delay in flowering time of the Mtphya mutants in VLD correlated with a very strong reduction in FTa1 transcript levels compared with wild type plants. The flowering time of Mtphya-1 ftal double mutants in VLD showed a weak additive effect in delaying flowering compared with the late flowering single mutants. Thus, although MtPHYA strongly positively regulates $F T a 1$ expression, MtPHYA might also regulate other flowering time targets.

Among the possible gene targets are MtE1L, MtFTb1, MtFTb2, MtFULa and MtFULb which were all induced by $L D$ photoperiods $[16,18,22]$ (this work) and were also decreased in the leaves of Mtphya-1 mutants in VLD and LD compared with wild type plants. MtFTb1 and $M t F T b 2$ function is not yet known, but their expression does not depend on MtFTa1 [16]. Interestingly, in addition, MtFULa and MtFULb transcript levels appeared to be more strongly reduced in the Mtphya-1 mutant than in the Mtfta1 mutant plants relative to wild type [22]. These results support the idea that there may also be FTa1-independent pathways influenced by
MtPHYA, MtFULs and MtFTbs in LD and VLDmediated promotion of flowering and/or primary stem elongation in Medicago.

$E 1$ is a major regulator of photoperiodic flowering in soybean and is regulated by soybean PHYA, raising the possibility that MtPHYA might regulate Medicago flowering time via the MtE1L homologue. Previous analysis of the Mte1l mutant and wild type plants indicated that $M t E 1 L$ promotes flowering in VLD [19]. Here, we showed that MtE1L transcript was photoperiodically regulated being more abundant in LD than in SD. MtE1L was reduced in Mtphya-1 mutants compared with wild type R108 under LD, SD and VLD conditions suggesting that $M t E 1 L$ is regulated by $M t P H Y A$. The double Mte1l Mtphya-1 mutants flowered at a similar time as the single Mtphya-1 mutants in VLD, suggesting that Mte1l and Mtphya-1 promote flowering in the same pathway in VLD. However, in contrast to soybean E1, MtE1L does not appear to have a major non-redundant role in promoting flowering in VLD in Medicago, because we only observed a moderate delay in flowering in the Mte1l mutants relative to R108 wild type plants.

The effect of Mtphya mutations on expression of candidate circadian clock has not been previously reported for pea or other temperate legumes. Here, most candidate circadian-clock related genes we analysed in Medicago did not vary in expression between the Mtphya-1 mutant and wild type at the time point analysed in VLD. However, MtGI transcript levels were modestly but consistently reduced in Mtphya-1 in both VLD and LD compared with R108. These results suggest that $M t P H Y A$ may regulate $M t G I$ expression during the relay of the LD photoperiod signal that accelerates flowering in Medicago.

\section{Conclusions}

Medicago is a long day plant, but the molecular basis of this photoperiodic regulation is still unclear because Medicago lacks a CO function, which has a central role in LD-mediated acceleration of flowering in Arabidopsis. Here, we demonstrate using reverse genetics that MtPHYA has an important role in LD photoperiodic control of flowering and aspects of plant architecture including primary stem elongation and seedling deetiolation under far-red wavelengths in Medicago. MtPHYA promotes the expression of LD-induced flowering time genes and modulates expression of clockrelated genes. In addition to MtFTa1, MtPHYA likely regulates other candidate targets in the LD and VLD floral induction pathway in Medicago. Thus, additional genomic and reverse genetic analyses could be carried out in the future to uncover the role of $M t G I, M t F T b 1$, MtFTb2, MtFULa and MtFULb in MtPHYA LD-induced flowering. 


\section{Methods}

\section{Plant materials and growth conditions}

Medicago truncatula (Medicago) wild-type R108_C3 (R108) [42] was used in this study. The R108 seeds were originally obtained from Dr. Pascal Ratet (Centre National de la Recherche Scientifique, Institut des Sciences du Vegetal, Gif-sur-Yvette, France). The seeds of the two Tnt1 insertion mutant alleles for MtPHYA, Mtphya-1 (NF1583) and Mtphya-2 (NF3601) (this work), the Tnt1 mutant for FTa1 (fta1, NF3307) [16] and the Tnt1 mutant for MtE1L (Mte1l, NF16583) [19] in the R108 background were obtained from the Noble Research Institute (Ardmore, OK, USA) [6].

Medicago plants were grown in controlled environments under $\sim 200 \mu \mathrm{M} \mathrm{m}^{-2} \mathrm{~s}^{-1}$ cool white fluorescent light at $22^{\circ} \mathrm{C}$ in long days (LD) (16 h light/8 h dark) or short days (SD) $(8 \mathrm{~h}$ light $/ 16 \mathrm{~h}$ dark) with or without prior vernalization of germinated seeds at $4{ }^{\circ} \mathrm{C}$ for 21 days, as previously described [12]. Flowering time was measured in either the total number of days after planting, or the total number of nodes along the primary axis at the time the first floral bud was observed by eye.

Homozygous Mtphya-1 plants were crossed with homozygous Mte1l plants and then bred and genotyped to identify Mtphya-1 Mte1l double F2 mutants. Similarly, homozygous Mtphya-1 was crossed with plants homozygous for fta1 to generate Mtphya-1 fta1 double mutants. Since, no double mutants were obtained from the segregating F2 plants, they were isolated from the F3 progeny of a Mtphya-1-homozygous/ fta1-heterozygous F2 plant. Genotyping was done using gene-specific and Tnt1 primers (see Additional file 2: Table S1).

\section{RNA extraction, CDNA synthesis and quantitative reverse transcriptase PCR (qRT-PCR)}

RNA extraction, cDNA synthesis using an oligo dT primer and qRT-PCR were carried out as previously described [12, 16, 18, 22]. Each data point represents the mean of three biological replicates harvested in parallel, with each replicate consisting of a pool of tissues from at least three independent plants at the 3-leaf stage of development ( 14-d-old), unless indicated otherwise. Leaf and shoot apices (hand-dissected by eye) were harvested separately. The identity of the PCR amplicons was checked by DNA sequencing. Gene expression was normalized to PROTEIN PHOSPHATASE 2A (PP2A) (Medtr6g084690). The relative gene expression was calculated based on the $2^{-\Delta \Delta \mathbf{C T}}$ method [43] with modifications [44] and normalised to the highest value as previously described [20]. Primers are shown in Additional file 2: Table S1.

The statistical testing for the gene expression data was performed using the one-way and the two-way analysis of variance (ANOVA) tests between the means $(\alpha=$
0.05). The Shapiro-Wilk normality assumption test was performed on all the data presented. Multiple pairwise comparisons adjusted for False Discovery Rate (FDR) was utilised to highlight statistically significant differences in the data presented.

\section{Hypocotyl elongation tests}

Seeds were scarified, sterilized and germinated in water overnight at $15^{\circ} \mathrm{C}$ with shaking in the dark. Germinated seeds were transferred into $1 / 2$ MS agar tubs with $8 \mathrm{~g} / \mathrm{L}$ Kalys agar, pH 5.8 and placed in the dark (D), continuous white light (WL), $\sim 100 \mu \mathrm{M} \mathrm{m}^{-2} \mathrm{~s}^{-1}$ or in far-red (FR) light at $22^{\circ} \mathrm{C}$ for three days. For the FR light setting, a spectral wavelength of $\sim 730 \mathrm{~nm}$ and intensity of $\sim 1.7 \mu \mathrm{M} \mathrm{m}^{-2} \mathrm{~s}^{-1}$ was used.

\section{Supplementary information}

Supplementary information accompanies this paper at https://doi.org/10. 1186/s12870-020-02540-y.

Additional file 1 Figure $\mathbf{S} \mathbf{1}$ Sequence alignment of PHYA-like proteins from temperate legumes and Arabidopsis.pdf. The deduced amino acid sequences were aligned using the MUSCLE plugin available in the Geneious software package [version 11.1.5 (http://www.geneious.com/)]. The domains highlighted include the N-terminal extension (NTE), Per-ArntSim (PAS), cGMP phosphodiesterase/ adenylate cyclase/FhIA (GAF), and phytochrome (PHY), which comprise the N-terminal photosensory core module. The C-terminal regulatory module consists of the PAS-related domain (PRD) containing two PAS repeats (PAS-A and PAS-B) and the histidine kinase-related domain (HKRD) [domains adopted from 27]. At: Arabidopsis thaliana, Ca: Cicer arietinum (chickpea), Lj: Lotus japonicas (Lotus), Mt: Medicago truncatula, Ps: Pisum sativum (pea), Tp: Trifolium pratense (red clover). Identical and similar residues are highlighted in black.

Additional file 2. Table S1. List of primers.pdf.

Abbreviations

LD: Long day; SD: Short day; VLD: Vernalized long day; VSD: Vernalized short day; qRT-PCR: quantitative reverse transcriptase PCR; FR: Far-red; WL: White light; D: Dark; FDR: False discovery rate; ANOVA: Analysis of variance

\section{Acknowledgements}

We thank Andrew Jiang, Julia Wei, Lulu Zhang and Geoffrey Thomson for technical assistance and comments on experiments. We thank Revel Drummond for providing and setting up the far-red LED arrays.

\section{Authors' contributions}

MJ and JP designed the study, performed the experiments, did the data analysis and wrote the paper. JW and KSM provided the Tnt 1 lines. All authors have read and approved the final manuscript.

\section{Funding}

This work was financially supported by a grant from the New Zealand Marsden Fund (www.royalsociety.org.nz/programmes/funds/marsden/) contract 14-UOA-125 awarded to JP. The fund was used to employ MJ as a Research Fellow and in the purchase of consumables and other materials used in the study. It was also used in the design of the study and collection, analysis, and interpretation of data and in writing the manuscript. The Tnt] line development and reverse genetics screenings were supported by the National Science Foundation, USA (DBI 0703285 and IOS-1127155), and Noble Research Institute, LLC. 


\section{Availability of data and materials}

All data generated or analysed during this study are included in this published article and its supplementary information files. The Medicago Tnt 1 insertion lines are available from the Noble Research Institute, LLC.

\section{Ethics approval and consent to participate}

Not applicable.

\section{Consent for publication}

Not applicable.

\section{Competing interests}

The authors declare that they have no competing interests.

\section{Author details}

${ }^{1}$ The Flowering Lab, School of Biological Sciences, University of Auckland, Auckland, New Zealand. ${ }^{2}$ Noble Research Institute, 2510 Sam Noble Parkway, Ardmore OK73401, USA.

Received: 31 March 2020 Accepted: 5 July 2020

Published online: 11 July 2020

\section{References}

1. Tadege M, Chen F, Murray J, Wen J, Ratet P, Udvardi M, Dixon R, Mysore K Control of vegetative to reproductive Phase transition improves biomass yield and simultaneously reduces lignin content in Medicago truncatula. Bioenerg Res. 2015;8(2):857-67.

2. Jung C, Muller AE. Flowering time control and applications in plant breeding. Trends Plant Sci. 2009:14(10):563-73.

3. Graham PH, Vance CP. Legumes: importance and constraints to greater use. Plant Physiol. 2003;131(3):872-7.

4. Weller $\mathrm{J}$, Ortega R. Genetic control of flowering time in legumes. Front Plant Sci. 2015;6(207):1-13.

5. Benlloch R, d'Erfurth I, Ferrandiz C, Cosson V, Beltran JP, Canas LA, Kondorosi A, Madueno F, Ratet P. Isolation of mtpim proves Tnt1 a useful reverse genetics tool in Medicago truncatula and uncovers new aspects of AP1-like functions in legumes. Plant Physiol. 2006;142(3):972-83.

6. Tadege M, Wang TL, Wen JQ, Ratet P, Mysore KS. Mutagenesis and beyond! Tools for understanding legume biology. Plant Physiol. 2009;151(3):978-84.

7. Young ND, Debelle F, Oldroyd GED, Geurts R, Cannon SB, Udvardi MK, Benedito VA, Mayer KFX, Gouzy J, Schoof H, et al. The Medicago genome provides insight into the evolution of rhizobial symbioses. Nature. 2011; 480(7378):520-4.

8. Putterill J, Zhang L, Yeoh C, Balcerowicz M, Jaudal M, Varkonyi Gasic E. FT genes and regulation of flowering in the legume Medicago truncatula. Functional Plant Biology. 2013;40:1199-207.

9. Jaudal M, Thomson G, Zhang L, Che C, Wen JS, Mysore K, Tadege M, Putterill J. Forward and reverse screens to identify genes that control vernalization and flowering time in Medicago truncatula. In: Fd B, editor. The Model Legume Medicago truncatula; 2020. p. 189-96.

10. Clarkson NM, Russell JS. Flowering responses to vernalisation and photoperiod in annual medics (Medicago spp). Aust J Agric Res. 1975;26(5): 831-8.

11. Wong ACS, Hecht VFG, Picard K, Diwadkar P, Laurie RE, Wen J, Mysore K, Macknight RC, Weller JL. Isolation and functional analysis of CONSTANS-LIKE genes suggests that a central role for CONSTANS in flowering time control is not evolutionarily conserved in Medicago truncatula. Front Plant Sci. 2014; $5: 486$

12. Zhang L, Jiang A, Thomson G, Kerr-Phillips M, Phan C, Krueger T, Jaudal M Wen J, Mysore KS, Putterill J. Overexpression of Medicago MtCDFd1_1 Causes Delayed Flowering in Medicago via Repression of MtFTa1 but Not MtCO-Like Genes. Front Plant Sci. 2019;10(1148):1-14.

13. Ridge S, Sussmilch FC, Hecht VF, Vander Schoor JK, Lee R, Aubert G, Burstin J, Macknight RC, Weller JL. Identification of LATE BLOOMER2 as a CYCLING DOF FACTOR homolog reveals conserved and divergent features of the flowering response to photoperiod in pea. Plant Cell. 2016;28(10):2545-59.

14. Hecht V, Knowles CL, Vander Schoor JK, Liew LC, Jones SE, Lambert MJM, Weller JL. Pea LATE BLOOMER1 is a GIGANTEA Ortholog with roles in photoperiodic flowering, Deetiolation, and transcriptional regulation of circadian clock gene homologs. Plant Physiol. 2007;144(2):648-61.
15. Liew LC, Hecht V, Laurie RE, Knowles CL, Vander Schoor JK, Macknight RC, Weller JL. DIE NEUTRALIS and LATE BLOOMER 1 contribute to regulation of the pea circadian clock. Plant Cell. 2009;21(10):3198-211.

16. Laurie RE, Diwadkar $P$, Jaudal M, Zhang LL, Hecht V, Wen JQ, Tadege M, Mysore KS, Putterill J, Weller JL, et al. The Medicago FLOWERING LOCUS T homolog, MtFTa1, is a key regulator of flowering time. Plant Physiol. 2011. 156(4):2207-24

17. Jaudal M, Yeoh CC, Zhang L, Stockum C, Mysore KS, Ratet P, Putterill J. Retroelement insertions at the Medicago FTa1 locus in spring mutants eliminate vernalisation but not long-day requirements for early flowering. Plant J. 2013:76(4):580-91.

18. Yeoh CC, Balcerowicz M, Zhang L, Jaudal M, Brocard L, Ratet P, Putterill J. Fine mapping links the FTa1 flowering time regulator to the dominant spring1 locus in Medicago. PLoS One. 2013;8(1):e53467.

19. Zhang $X$, Zhai H, Wang Y, Tian X, Zhang Y, Wu H, Lü S, Yang G, Li Y, Wang $L$, et al. Functional conservation and diversification of the soybean maturity gene E1 and its homologs in legumes. Sci Rep. 2016;6:29548.

20. Jaudal M, Zhang L, Che C, Li G, Tang Y, Wen J, Mysore KS, Putterill J. A SOC1-like gene MtSOC1a promotes flowering and primary stem elongation in Medicago. J Exp Bot. 2018;69(20):4867-80.

21. Fudge JB, Lee RH, Laurie RE, Mysore KS, Wen J, Weller JL, Macknight RC. Medicago truncatula SOC1 Genes Are Up-regulated by Environmental Cues That Promote Flowering. Front Plant Sci. 2018;9(496):1-11.

22. Jaudal M, Zhang L, Che C, Putterill J. Three Medicago MtFUL genes have distinct and overlapping expression patterns during vegetative and reproductive development and 35S:MtFULb accelerates flowering and causes a terminal flower phenotype in Arabidopsis. Front Genet. 2015;6(50): $1-14$.

23. Jaudal M, Zhang L, Che C, Hurley DG, Thomson G, Wen J, Mysore KS, Putterill J. MtVRN2 is a Polycomb VRN2-like gene which represses the transition to flowering in the model legume Medicago truncatula. Plant J. 2016:86(2):145-60.

24. Jaudal M, Monash J, Zhang L, Wen J, Mysore KS, Macknight R, Putterill J. Overexpression of Medicago SVP genes causes floral defects and delayed flowering in Arabidopsis but only affects floral development in Medicago. J Exp Bot. 2014;65(2):429-42.

25. Hoecker $U$. The activities of the E3 ubiquitin ligase COP1/SPA, a key repressor in light signaling. Curr Opin Plant Biol. 2017;37:63-9.

26. Podolec R, Ulm R. Photoreceptor-mediated regulation of the COP1/SPA E3 ubiquitin ligase. Curr Opin Plant Biol. 2018;45:18-25.

27. Li J, Li G, Wang H, Wang Deng X. Phytochrome signaling mechanisms. Arabidopsis Book. 2011;9:e0148.

28. Valverde F, Mouradov A, Soppe W, Ravenscroft D, Samach A, Coupland G. Photoreceptor regulation of CONSTANS protein in photoperiodic flowering. Science. 2004:303(5660):1003-6.

29. Weller JL, Murfet IC, Reid JB. Pea mutants with reduced sensitivity to far-red light define an important role for Phytochrome a in day-length detection. Plant Physiol. 1997;114(4):1225-36.

30. Weller JL, Batge SL, Smith JJ, Kerckhoffs LHJ, Sineshchekov VA, Murfet IC, Reid JB. A dominant mutation in the pea PHYA gene confers enhanced responses to light and impairs the light-dependent degradation of Phytochrome a. Plant Physiol. 2004;135(4):2186-95.

31. Zhang Y, Sun J, Xia H, Zhao C, Hou L, Wang B, Li A, Chen M, Zhao S, Wang $X$. Characterization of peanut phytochromes and their possible regulating roles in early peanut pod development. PLoS One. 2018;13(5):-e0198041.

32. Hecht V, Foucher F, Ferrandiz C, Macknight R, Navarro C, Morin J, Vardy ME, Ellis N, Beltran JP, Rameau C, et al. Conservation of Arabidopsis flowering genes in model legumes. Plant Physiol. 2005;137(4):1420-34.

33. Hennig L, Büche C, Eichenberg K, Schäfer E. Dynamic properties of endogenous Phytochrome a in Arabidopsis seedlings. Plant Physiol. 1999; 121(2):571-7.

34. Weller JL, Beauchamp N, Kerckhoffs LHJ, Platten JD, Reid JB. Interaction of phytochromes $\mathrm{a}$ and $\mathrm{B}$ in the control of de-etiolation and flowering in pea. Plant J. 2001:26(3):283-94.

35. Franklin KA, Quail PH. Phytochrome functions in Arabidopsis development. J Exp Bot. 2010;61(1):11-24.

36. Liu B, Kanazawa A, Matsumura H, Takahashi R, Harada K, Abe J. Genetic redundancy in soybean Photoresponses associated with duplication of the Phytochrome a gene. Genetics. 2008;180(2):995-1007.

37. Watanabe $S$, Hideshima $R$, Xia Z, Tsubokura $Y$, Sato $S$, Nakamoto $Y$, Yamanaka N, Takahashi R, Ishimoto M, Anai T, et al. Map-based cloning of 
the gene associated with the soybean maturity locus E3. Genetics. 2009; 182(4):1251-62.

38. Weller JL, Vander Schoor JK, Perez-Wright EC, Hecht V, González AM, Capel C, Yuste-Lisbona FJ, Lozano R, Santalla M. Parallel origins of photoperiod adaptation following dual domestications of common bean. J Exp Bot. 2019;70(4):1209-19.

39. Parks BM. Quail PH: hy8, a new class of arabidopsis long hypocotyl mutants deficient in functional phytochrome a. Plant Cell. 1993;5(1):39-48.

40. van Tuinen A, Kerckhoffs LHJ, Nagatani A, Kendrick RE, Koornneef M. Far-red light-insensitive, phytochrome A-deficient mutants of tomato. Mol Gen Genet. 1995;246(2):133-41.

41. Kong F, Liu B, Xia Z, Sato S, Kim BM, Watanabe S, Yamada T, Tabata S, Kanazawa A, Harada K, et al. Two coordinately regulated homologs of FLOWERING LOCUS T are involved in the control of photoperiodic flowering in soybean. Plant Physiol. 2010;154(3):1220-31.

42. Trinh $\mathrm{HT}$, Ratet $\mathrm{P}$, Kondorosi $\mathrm{E}$, Durand $\mathrm{P}$, Kamaté $\mathrm{K}$, Bauer $\mathrm{P}$, Kondorosi $\mathrm{A}$. Rapid and efficient transformation of diploid Medicago truncatula and Medicago sativa ssp. falcata lines improved in somatic embryogenesis. Plant Cell Rep. 1998;17(5):345-55.

43. Livak KJ, Schmittgen TD. Analysis of relative gene expression data using real-time quantitative PCR and the 2-[Delta][Delta] CT method. Methods. 2001:25(4):402-8.

44. Bookout AL, Mangelsdorf DJ. Quantitative real-time PCR protocol for analysis of nuclear receptor signaling pathways. Nucl Recept Signal. 2003;1:e012.

\section{Publisher's Note}

Springer Nature remains neutral with regard to jurisdictional claims in published maps and institutional affiliations.

Ready to submit your research? Choose BMC and benefit from:

- fast, convenient online submission

- thorough peer review by experienced researchers in your field

- rapid publication on acceptance

- support for research data, including large and complex data types

- gold Open Access which fosters wider collaboration and increased citations

- maximum visibility for your research: over $100 \mathrm{M}$ website views per year

At $\mathrm{BMC}$, research is always in progress.

Learn more biomedcentral.com/submissions 